\title{
MODELING OF THE DIFFUSION PHENOMENON IN MEDIA WITH MEMBRANES BY GENERALIZED DIFFUSION PROCESSES
}

\author{
Bohdan Kopytko ${ }^{1}$, Roman Shevchuk ${ }^{2}$ \\ ${ }^{I}$ Institute of Mathematics, Czestochowa University of Technology \\ Czestochowa, Poland \\ ${ }^{2}$ Vasyl Stefanyk Precarpathian National University \\ Ivano-Frankivsk, Ukraine \\ bohdan.kopytko@im.pcz.pl,r.v.shevchuk@gmail.com
}

\begin{abstract}
Generalized diffusion processes can serve as mathematical models of physical phenomenon of diffusion in media with the membranes on some fixed surfaces. Qualitatively, such processes differ from the ordinary diffusion process only at the points of location of the membrane, whose type is specified by the corresponding variants of the general Feller-Wentzell boundary condition (one-dimensional case) and the Wentzell condition (multidimensional case). An important problem in the theory of diffusion processes is the development of methods of construction of the process by the given diffusion characteristics: the diffusion matrix and the drift vector, and in the case of the state space with the membrane, by the boundary conditions additionally specified on it. One of such problems, which leads to a generalized diffusion process, is the object of study in this chapter. It presents the results obtained by the authors concerning the question of construction of an integral representation of a two-parameter Feller semigroup associated with a one-dimensional inhomogeneous diffusion process with a membrane whose properties are described by a nonlocal Feller-Wentzell boundary condition given at it the point of its location.
\end{abstract}

Keywords: generalized diffusion process, Feller semigroup, parabolic potential, parabolic conjugation problem, nonlocal condition

\section{Introduction}

The aim of this chapter is to construct and study some mathematical models of diffusion phenomenon in media with translucent membranes. It is well known (see, for instance, $[1,2]$ ) that the ordinary diffusion is modeled in mathematics by the concept of the diffusion process. That is the Markov process which has local characteristics of motion: the drift vector, which represents the macroscopic speed of the medium motion at a given point at a given moment of time and the diffusion matrix, which characterizes the intensity of the heat motion of molecules also at a given space point and at a given moment of time. According to [3, 4] the specific type of the membranes located on some surface for the ordinary diffusion process arises in situation when this process is perturbed by vector field with a structure of $\delta$-function on this surface. Such a perturbance leads to the generalized diffusion 
process for which the diffusion matrix remains the same as for the not perturbed process, and the drift vector is the sum of those for the not perturbed process and the generalized function. Furthermore, the sample paths of this new process also remain continuous. The method of construction of the desired process is analytic. It is based on the semigroup theory and the classical potential theory. The generalized diffusion process constructed in this way is an example of the continuous Feller process which describes the diffusion phenomenon in a medium with a translucent membrane on the given surface. This process is also called the diffusion process with partial reflection at some surface.

The membranes of the more difficult type, and thus the generalized diffusion processes corresponding to them appear in the so-called problem of pasting together diffusion processes in the finite dimensional Euclidean space $[4,5]$. The application of the analytical approach to the solution of this problem necessitates the study of the corresponding conjugation problem for a linear parabolic equation of a second order, where one of two conjugation conditions given on a fixed hypersurface on which the membrane is placed corresponds to the general Wentzell boundary condition for multi-dimensional diffusion processes [6] (in the one-dimensional case it is the Feller-Wentzell condition [7-9]). The Feller semigroup defined using the solution of the parabolic conjugation problem describes the desired process. The development of this approach to construction of generalized diffusion processes and the study of their properties by methods of potential theory is partially reflected in works $[4,5]$ we have already mentioned (see also references given there). Moreover, the method of solving the parabolic conjugation problems, mentioned above, was applied in [10] in the problem of high-energy astrophysics to construct the classical solution of the special case of a non-stationary kinetic equation describing the acceleration of charged particles in the vicinity of strong shock waves. We also mention works $[11,12]$, where the one-parameter Feller semigroups associated with diffusion processes in a domain with different variants of the Wentzell boundary condition were constructed by methods of functional analysis. Here we can also include the work [9] in which the problem of pasting together two homogeneous diffusion processes on a line was studied.

The generalized diffusion processes constructed by the analytic method can be characterized using the $\mathrm{W}$-functional technique as weak solutions of the stochastic differential equations [3-5]. In the most general case, the problem of pasting together diffusion processes in martingale formulation was considered in work [13]. In [14] it is shown that the generalized diffusion processes can be obtained as the weak limits of a sequence of diffusion processes. In special cases (see [15-17]) the generalized diffusion was constructed by the direct probabilistic method, that is, using the strong solutions of the corresponding stochastic differential equations. Besides, in one-dimensional case there were considered the equations which are more difficult with respect to the assumptions on the drift coefficient or on the behavior of process at the boundary of the domain (see [18-21]), while in a multidimensional situation the probabilistic approach is most fully investigated only for the diffusion processes with pure reflection (see [22] and references given there). 
In this chapter we restrict our attention to the problem of pasting together two diffusion processes in a one-dimensional case. Most of the material given here is from $[23,24]$.

Now let $D_{i}=\left\{x \in \mathbb{R}:(-1)^{i} x>0\right\}, i=1,2$, be the two domains on the line $\mathbb{R}$ with the common boundary $S=\{0\}$ and the closures $\bar{D}_{i}=D_{i} \cup\{0\}$, and let $T>0$ be fixed. If $\Gamma$ is $\bar{D}_{i}$ or $\mathbb{R}$, then we denote by $C_{b}(\Gamma)$ the Banach space of all real-valued bounded and continuous on $\Gamma$ functions $\varphi$ with the norm

$$
\|\varphi\|=\sup _{x \in \Gamma}|\varphi(x)|,
$$

and by $C_{2}(\Gamma)$ the set of all functions $\varphi$ that are bounded and uniformly continuous on $\Gamma$ together with their first- and second-order derivatives. Let $\varphi_{i}$ be the restriction of any function $\varphi \in C_{b}(\mathbb{R})$ to $D_{i}$.

Assume that an inhomogeneous diffusion process is given in $D_{i}, i=1,2$, and it is generated by a second-order differential operator $A_{s}^{(i)}, s \in[0, T]$, that acts on $C_{2}\left(\bar{D}_{i}\right)$ :

$$
A_{s}^{(i)} \varphi_{i}(x)=\frac{1}{2} b_{i}(s, x) \frac{d^{2} \varphi_{i}(x)}{d x^{2}}+a_{i}(s, x) \frac{d \varphi_{i}(x)}{d x}, \quad i=1,2,
$$

where $b_{i}(s, x)$ and $a_{i}(s, x)$ are real-valued continuous bounded functions in the domain $(s, x) \in[0, T] \times \bar{D}_{i}$ and $b_{i}(s, x) \geq 0$.

Denote by $C_{2,0}(\mathbb{R})$ the subset of $C_{b}(\mathbb{R})$ consisting of all functions $\varphi(x)$ such that $\varphi_{i} \in C_{2}\left(\bar{D}_{i}\right)$ for $i=1,2, A_{s}^{(1)} \varphi_{1}(0)=A_{s}^{(2)} \varphi_{2}(0)$, and define the operator $A_{s}$, acting on $C_{2,0}(\mathbb{R})$ as follows:

$$
A_{s} \varphi(x)= \begin{cases}A_{s}^{(1)} \varphi_{1}(x), & x \in \bar{D}_{1}, \\ A_{s}^{(2)} \varphi_{2}(x), & x \in \bar{D}_{2} .\end{cases}
$$

Assume also that there is given the Feller-Wentzell conjugation operator which acts on the function $\varphi \in C_{2,0}(\mathbb{R})$ by the formula

$$
\begin{aligned}
L_{s} \varphi(0) & =r(s) A_{s} \varphi(0)+q_{1}(s) \varphi^{\prime}(0-)-q_{2}(s) \varphi^{\prime}(0+)+\gamma(s) \varphi(0)+ \\
& +\int_{D_{1} \cup D_{2}}[\varphi(0)-\varphi(y)] \mu(s, d y)=0, s \in[0, T],
\end{aligned}
$$

where the coefficients $r, q_{1}, q_{2}, \gamma$ and the measure $\mu$ satisfy the following conditions: 
1.1. The functions $r(s), q_{1}(s), q_{2}(s), \gamma(s)$ are nonnegative and continuous on $[0, T]$.

1.2. For a fixed $s, \mu(s, \cdot)$ is a nonnegative measure on $D_{1} \cup D_{2}$ such that for any $\delta>0$ and for all functions $f \in C_{b}(\mathbb{R})$ the integrals

$$
\int_{D_{i}^{\delta}} y f(y) \mu(s, d y), \quad \int_{D_{i} \backslash D_{i}^{\delta}} f(y) \mu(s, d y), \quad i=1,2,
$$

are continuous on $[0, T]$ as functions of $s$, where $D_{i}^{\delta}=\left\{y \in D_{i}\right.$ : $|y|<\delta\}, i=1,2$.

1.3. $r(s)+q_{1}(s)+q_{2}(s)+\gamma(s)+\mu\left(s, D_{1} \cup D_{2}\right)>0$ for all $s \in[0, T]$.

It is known (see [9]) that the operator $L_{s}$ defines the most general condition of conjugation, which is of the form

$$
L_{s} \varphi(0)=0, s \in[0, T],
$$

and which restricts the differential operator $A_{s}$ in (2) to an infinitesimal generator of a Feller semigroup in the space of bounded continuous functions. Thus, the condition (4) is the general Feller-Wentzell conjugation condition by which one can describe all the possible types of behavior of a diffusing particle at the time when it reaches the point zero. These types of behavior are as follows: the sticking (viscosity) $\left(r>0, q_{1} \equiv q_{2} \equiv \gamma \equiv \mu \equiv 0\right)$, the partial reflection $\left(q_{1}+q_{2}>0, r \equiv \gamma \equiv \mu \equiv 0\right)$, the absorption $\left(\gamma>0, r \equiv q_{1} \equiv q_{2} \equiv \mu \equiv 0\right)$, the jump $\left(\mu>0, r \equiv q_{1} \equiv q_{2} \equiv \gamma \equiv 0\right)$ as well as their combinations (the linear combinations of the corresponding boundary conditions).

The problem is to clarify the question about the existence of a two-parameter semigroup of operators $T_{s, t}, 0 \leq s<t \leq T$, describing the sufficiently general classes of inhomogeneous Feller processes in $\mathbb{R}$ such that in the domains $D_{1}$ and $D_{2}$ they coincide with the given diffusion processes generated by the operators $A_{s}^{(1)}$ and $A_{s}^{(2)}$, respectively, and their behavior at the point zero is determined by the corresponding variants to these classes of the general Feller-Wentzell conjugation condition (4). This is a so-called problem of pasting together two diffusion processes on a line.

The investigation of the problem formulated above is performed by the analytic method. Such an approach allows us to define the desired operator family using the solution of the corresponding problem of conjugation for a linear parabolic equation of the second order with variable coefficients, discontinuous at the zero point. This problem is to find a function $u(s, x, t)=T_{s, t} \varphi(x)$ satisfying the following conditions: 


$$
\begin{gathered}
\frac{\partial u(s, x, t)}{\partial s}+A_{s}^{(i)} u(s, x, t)=0, \quad 0 \leq s<t \leq T, x \in D_{i}, i=1,2, \\
\lim _{s \uparrow t} u(s, x, t)=\varphi(x), \quad x \in \mathbb{R}, \\
u(s, 0-, t)=u(s, 0+, t), \quad 0 \leq s<t \leq T, \\
L_{s} u(s, 0, t)=0, \quad 0 \leq s<t \leq T,
\end{gathered}
$$

where $\varphi \in C_{b}(\mathbb{R})$ is a given function. As we see, the condition (7) in the problem (5)-(8) is the consequence of the Feller property of the desired semigroup $T_{s, t}$, and the equality (8) corresponds to the general Feller-Wentzell conjugation condition (3), (4). In comparison with classical cases, the peculiarity of the conjugation condition (8) is that it is nonlocal, furthermore, the measure $\mu$ in the integral term of (3) can be infinite.

To solve the problem (5)-(8) in general, we consider three possible variants of conjugation condition (8). The first two variants of the condition (8) are transversal (it includes the local terms of the orders higher than the order of the nonlocal one). In the first one $r \equiv 0, q_{1}+q_{2}>0$ and in the second one, the conjugation condition (8) may include all its terms. These cases are studied, respectively, in Sections 3 and 4 of the present chapter. In the last variant, the condition (8) is non-transversal, i.e., $r \equiv q_{1} \equiv q_{2} \equiv 0$. This situation is briefly considered in Section 5 .

\section{Preliminaries}

Consider the Kolmogorov backward equations (5) $(i=1,2)$. Assume that their coefficients $a_{i}(s, x)$ and $b_{i}(s, x)$ are defined on $[0, T] \times \mathbb{R}$ and satisfy the following conditions:

2.1. There exist constants $B$ and $b$ such that $0<b \leq b_{i}(s, x) \leq B$ for all $(s, x) \in[0, T] \times \mathbb{R}$.

2.2. The functions $a_{i}(s, x)$ are bounded on $[0, T] \times \mathbb{R}$.

2.3. For all $s, s^{\prime} \in[0, T], x, x^{\prime} \in \mathbb{R}$ the next inequalities hold:

$$
\begin{aligned}
& \left|b_{i}(s, x)-b_{i}\left(s^{\prime}, x^{\prime}\right)\right| \leq c\left(\left|s-s^{\prime}\right|^{\frac{\alpha}{2}}+\left|x-x^{\prime}\right|^{\alpha}\right), \\
& \left|a_{i}(s, x)-a_{i}\left(s^{\prime}, x^{\prime}\right)\right| \leq c\left(\left|s-s^{\prime}\right|^{\frac{\alpha}{2}}+\left|x-x^{\prime}\right|^{\alpha}\right),
\end{aligned}
$$

where $c$ and $\alpha$ are positive constants, $0<\alpha<1$. 
From the conditions 2.1-2.3 there follows the existence of the fundamental solutions of equations (5) in the domain $[0, T] \times \mathbb{R}$, i.e., the existence of the functions $G_{i}(s, x, t, y)$ defined for $0 \leq s<t \leq T, x, y \in \mathbb{R}$ such that:

a) they are jointly continuous;

b) for fixed $t \in(0, T], y \in \mathbb{R}$ they satisfy equations (5);

c) for any function $\varphi \in C_{b}(\mathbb{R})$ and any $t \in(0, T], x \in \mathbb{R}$

$$
\lim _{s \uparrow t} \int_{\mathbb{R}} G_{i}(s, x, t, y) \varphi(y) d y=\varphi(x)
$$

Furthermore, in the domain $0 \leq s<t \leq T, x, y \in \mathbb{R}$ the following estimations for permissible derivatives of the functions $G_{i}(s, x, t, y)$ hold:

$$
\left|D_{s}^{r} D_{x}^{p} G_{i}(s, x, t, y)\right| \leq c(t-s)^{-\frac{1+2 r+p}{2}} \exp \left\{-h \frac{(y-x)^{2}}{t-s}\right\},
$$

where $r$ and $p$ are the nonnegative integers such that $2 r+p \leq 2 ; D_{s}^{r}$ is the partial derivative with respect to $s$ of order $r ; D_{x}^{p}$ is the partial derivative with respect to $x$ of order $p ; c, h$ are various positive constants. Recall also that $G_{i}(s, x, t, y)$, $i=1,2$, are represented as

$$
G_{i}(s, x, t, y)=Z_{i 0}(s, x, t, y)+Z_{i 1}(s, x, t, y)
$$

where

$$
Z_{i 0}(s, x, t, y)=\left[2 \pi b_{i}(t, y)(t-s)\right]^{-\frac{1}{2}} \exp \left\{-\frac{(y-x)^{2}}{2 b_{i}(t, y)(t-s)}\right\}
$$

and the functions $Z_{i 1}(s, x, t, y)$ satisfy the inequalities

$$
\left|D_{s}^{r} D_{x}^{p} Z_{i 1}(s, x, t, y)\right| \leq c(t-s)^{-\frac{1+2 r+p-\alpha}{2}} \exp \left\{-h \frac{(y-x)^{2}}{t-s}\right\}
$$

where $0 \leq s<t \leq T, x, y \in \mathbb{R}, 2 r+p \leq 2, \alpha$ is the constant from 2.3.

We mention the following relations (see [4, p. 53]) valid for $0 \leq s<t \leq T$, $i=1,2$ :

$$
\int_{\mathbb{R}}(y-x) G_{i}(s, x, t, y) d y=\int_{s}^{t} d \tau \int_{\mathbb{R}} G_{i}(s, x, \tau, z) a_{i}(\tau, z) d z,
$$




$$
\begin{aligned}
& \int_{\mathbb{R}}(y-x)^{2} G_{i}(s, x, t, y) d y=\int_{s}^{t} d \tau \int_{\mathbb{R}} G_{i}(s, x, \tau, z) b_{i}(\tau, z) d z+ \\
& \quad+2 \int_{s}^{t} d \tau \int_{\mathbb{R}} G_{i}(s, x, \tau, z) a_{i}(\tau, z)(z-x) d z .
\end{aligned}
$$

Given the fundamental solution $G_{i}, i=1,2$, we define the parabolic potentials that will be used to solve the problem (5)-(8), namely the Poisson potential

$$
u_{i 0}(s, x, t)=\int_{\mathbb{R}} G_{i}(s, x, t, y) \varphi(y) d y, 0 \leq s<t \leq T, x \in \mathbb{R},
$$

and the simple-layer potential

$$
u_{i 1}(s, x, t)=\int_{s}^{t} G_{i}(s, x, \tau, 0) V_{i}(\tau, t, \varphi) d \tau
$$

where $\varphi$ is the function from (6) and $V_{i}(s, t, \varphi), i=1,2$, are some functions, continuous in $0 \leq s \leq t \leq T$. We mention the following properties of potentials $u_{i 0}$ and $u_{i 1}$ (see, e.g., $\left.[1,4,25]\right)$ :

a) the functions $u_{i 0}, u_{i 1}, i=1,2$, are continuous in $0 \leq s<t \leq T, x \in \mathbb{R}$, bounded with respect to $x$, satisfy the equations (5) in the domains $(s, x) \in[0, t) \times \mathbb{R}$, $(s, x) \in[0, t) \times\left(D_{1} \cup D_{2}\right)$ respectively, and satisfy the initial conditions

$$
\lim _{s \uparrow t} u_{i 0}(s, x, t)=\varphi(x), \lim _{s \uparrow t} u_{i 1}(s, x, t)=0, x \in \mathbb{R}
$$

b) for the potential $u_{i 0}, i=1,2$, the following estimations are valid:

$$
\left|D_{s}^{r} D_{x}^{p} u_{i 0}(s, x, t)\right| \leq c\|\varphi\|(t-s)^{-\frac{2 r+p}{2}}
$$

c) the potential $u_{i 1}, i=1,2$, satisfies the relation

$$
\frac{\partial u_{i 1}(s, 0 \mp, t)}{\partial x}= \pm \frac{V_{i}(s, t, \varphi)}{b_{i}(s, 0)}+\int_{s}^{t} \frac{\partial Z_{i 1}(s, 0, \tau, 0)}{\partial x} V_{i}(\tau, t, \varphi) d \tau
$$

that is often called the formula on a jump for a simple-layer potential.

Note that the properties of the simple-layer potential mentioned above remain valid under somewhat more general assumptions on the functions $V_{i}, i=1,2$, in particular, when

$$
\left|V_{i}(s, t, \varphi)\right| \leq c(t-s)^{-1+\varepsilon},
$$

for any $\varepsilon>0($ see $[26$, Ch.V, Sec. 2, 3]). 


\section{Process with partial reflection, absorption and jump properties}

In this section we solve the problem (5)-(8) in the special case when the coefficients of $L_{s}, s \in[0, T]$, satisfy the condition

I. $r(s)=0, \quad q_{1}(s)+q_{2}(s)>0$ for all $s \in[0, T]$.

Theorem 3.1. Assume that the conditions I, 1.1, 1.2 and 2.1-2.3 hold. Then for every function $\varphi \in C_{b}(\mathbb{R})$ the problem (5)-(8) has a unique solution

$$
u(s, x, t) \in C^{1,2}\left([0, t) \times D_{1} \cup D_{2}\right) \cap C([0, t] \times \mathbb{R}) .
$$

Furthermore, this solution satisfies the inequality

$$
|u(s, x, t)| \leq c\|\varphi\|, 0 \leq s<t \leq T,
$$

and has the form

$$
u(s, x, t)=\int_{\mathbb{R}} G_{i}(s, x, t, y) \varphi(y) d y+\int_{s}^{t} G_{i}(s, x, \tau, 0) V_{i}(\tau, t, \varphi) d \tau,
$$

where a pair of functions $\left(V_{1}, V_{2}\right)$ is a solution of some system of Volterra integral equations of the second kind.

Proof. We find a solution of the problem (5)-(8) of the form (20) with the unknown functions $V_{i}, i=1,2$, that will be determined from the conjugation conditions (7), (8). If we substitute the expression (20) for $u(s, x, t)$ into (7), (8) and use therewith the formula on the jump for a simple-layer potential (17), we obtain the following system of integral equations for $V_{i}$

$$
\begin{aligned}
& \sum_{i=1}^{2} \int_{s}^{t}(-1)^{i} G_{i}(s, 0, \tau, 0) V_{i}(\tau, t, \varphi) d \tau=\Upsilon(s, t, \varphi), \\
& \sum_{i=1}^{2}\left(\frac{q_{i}(s)}{b_{i}(s, 0)} V_{i}(s, t, \varphi)-\int_{s}^{t} \bar{K}_{i}(s, \tau) V_{i}(\tau, t, \varphi) d \tau\right)=\Psi(s, t, \varphi),
\end{aligned}
$$

where

$$
\begin{aligned}
& \Upsilon(s, t, \varphi)=u_{10}(s, 0, t)-u_{20}(s, 0, t), \Psi(s, t, \varphi)=\sum_{i=1}^{2}\left((-1)^{i} q_{i}(s) \frac{\partial u_{i 0}(s, 0, t)}{\partial x}-\right. \\
& \left.-\frac{\gamma(s)}{2} u_{i 0}(s, 0, t)-\int_{D_{i}}\left[u_{i 0}(s, 0, t)-u_{i 0}(s, y, t)\right] \mu(s, d y)\right), \bar{K}_{i}(s, \tau)=(-1)^{i} q_{i}(s) \times \\
& \times \frac{\partial Z_{i 1}(s, 0, \tau, 0)}{\partial x}-\frac{\gamma(s)}{2} G_{i}(s, 0, \tau, 0)-\int_{D_{i}}\left[G_{i}(s, 0, \tau, 0)-G_{i}(s, y, \tau, 0)\right] \mu(s, d y) .
\end{aligned}
$$


The two equations in (21) are the Volterra integral equations of the first and second kinds, respectively. We have to reduce the first one to an equivalent Volterra integral equation of the second kind. To do this, we define the Holmgren's operator

$$
\mathcal{E}(s, t) \Upsilon=\sqrt{\frac{2}{\pi}} \frac{\partial}{\partial s} \int_{s}^{t}(\rho-s)^{-\frac{1}{2}} \Upsilon(\rho, t, \varphi) d \rho, \quad 0 \leq s<t \leq T,
$$

and apply it to both sides of this equation. After some direct simplifications, we obtain

$$
\sum_{i=1}^{2}(-1)^{i}\left(\int_{s}^{t} \tilde{K}_{i}(s, \tau) V_{i}(\tau, t, \varphi) d \tau-\frac{V_{i}(s, t, \varphi)}{\sqrt{b_{i}(s, 0)}}\right)=\Phi(s, t, \varphi)
$$

where

$$
\begin{aligned}
& \tilde{K}_{i}(s, \tau)=\sqrt{\frac{2}{\pi}} \frac{\partial}{\partial s} \int_{s}^{\tau}(\rho-s)^{-\frac{1}{2}} Z_{i 1}(\rho, 0, \tau, 0) d \rho= \\
& =\frac{1}{\sqrt{2 \pi}} \int_{s}^{\tau}(\rho-s)^{-\frac{3}{2}}\left(Z_{i 1}(\rho, 0, \tau, 0)-Z_{i 1}(s, 0, \tau, 0)\right) d \rho-\sqrt{\frac{2}{\pi}} Z_{i 1}(s, 0, \tau, 0)(\tau-s)^{-\frac{1}{2}}, \\
& \Phi(s, t, \varphi)=\frac{1}{\sqrt{2 \pi}} \int_{s}^{t}(\rho-s)^{-\frac{3}{2}}(\Upsilon(\rho, t, \varphi)-\Upsilon(s, t, \varphi)) d \rho-\sqrt{\frac{2}{\pi}} \Upsilon(s, t, \varphi)(t-s)^{-\frac{1}{2}} .
\end{aligned}
$$

Next, writing the equation (23) instead of the first equation in (21), we find that the system (21) can be replaced by an equivalent system of Volterra integral equations of the second kind:

$$
V_{i}(s, t, \varphi)=\sum_{j=1}^{2} \int_{s}^{t} K_{i j}(s, \tau) V_{j}(\tau, t, \varphi) d \tau+\Psi_{i}(s, t, \varphi), \quad i=1,2,
$$

where

$$
\begin{aligned}
& K_{i j}(s, \tau)=d_{i}(s)\left(\bar{K}_{j}(s, \tau)+(-1)^{i+j} \frac{q_{3-i}(s)}{\sqrt{b_{3-i}(s, 0)}} \tilde{K}_{j}(s, \tau)\right), \\
& \Psi_{i}(s, t, \varphi)=d_{i}(s)\left(\Psi(s, t, \varphi)+(-1)^{i-1} \frac{q_{3-i}(s)}{\sqrt{b_{3-i}(s, 0)}} \Phi(s, t, \varphi)\right), \\
& d_{i}(s)=\frac{b_{i}(s, 0) \sqrt{b_{3-i}(s, 0)}}{q_{1} \sqrt{b_{2}(s, 0)}+q_{2} \sqrt{b_{1}(s, 0)}} .
\end{aligned}
$$

Let us show that the system of equations (24) has a solution which can be obtained by the method of successive approximations 


$$
V_{i}(s, t, \varphi)=\sum_{n=0}^{\infty} V_{i}^{(n)}(s, t, \varphi), \quad 0 \leq s<t \leq T, \quad i=1,2
$$

where

$$
V_{i}^{(0)}(s, t, \varphi)=\Psi_{i}(s, t, \varphi), \quad V_{i}^{(n)}(s, t, \varphi)=\sum_{j=1}^{2} \int_{s}^{t} K_{i j}(s, \tau) V_{j}^{(n-1)}(\tau, t, \varphi) d \tau, \quad n=1,2, \ldots
$$

To this end, we first have to estimate the functions $\Psi_{i}$ and the kernels $K_{i j}$ in (24).

The estimation for $\Psi_{i}$ can be easily established by using the inequalities (16). Consider, for example, the integral (this is the last term of the formula for the function $\Psi$ ):

$$
\begin{aligned}
& I_{i}(s, t, \varphi)=\int_{D_{i}}\left[u_{i 0}(s, 0, t)-u_{i 0}(s, y, t)\right] \mu(s, d y)= \\
& =\int_{D_{i}^{1}}\left[u_{i 0}(s, 0, t)-u_{i 0}(s, y, t)\right] \mu(s, d y)+\int_{D_{i} \backslash_{D_{i}^{1}}^{1}}\left[u_{i 0}(s, 0, t)-u_{i 0}(s, y, t)\right] \mu(s, d y) .
\end{aligned}
$$

Denote by $I_{i 1}$ and $I_{i 2}$ the first and second terms in the expression (26), respectively. In order to estimate $I_{i 1}$, first, we apply the Lagrange formula to the integrand $u_{i 0}(s, 0, t)-u_{i 0}(s, y, t)$

$$
u_{i 0}(s, 0, t)-u_{i 0}(s, y, t)=\left.\frac{\partial u_{i 0}(s, x, t)}{\partial x}\right|_{x=\theta y} \cdot y
$$

where $\theta$ is some real number from the interval $(0,1)$. Then, using the inequality (16) when $r=0$ and $p=1$, we find that

$$
\left|I_{i 1}(s, t, \varphi)\right| \leq c\|\varphi\|(t-s)^{-\frac{1}{2}} .
$$

It is clear that the same estimation is also valid for the integral $I_{i 2}$. Estimating all the remaining terms in the expression for $\Psi_{i}$, we conclude that

$$
\left|\Psi_{i}(s, t, \varphi)\right| \leq c\|\varphi\|(t-s)^{-\frac{1}{2}} .
$$

We proceed to estimate the kernels $K_{i j}(s, \tau), i=1,2, j=1,2$, in (24). For this purpose, in the expression for $\bar{K}_{j}$ we take the component

$$
N_{j, \delta}(s, \tau)=\int_{D_{j}^{\delta}}\left[Z_{j 0}(s, 0, \tau, 0)-Z_{j 0}(s, y, \tau, 0)\right] \mu(s, d y)
$$


(here $\delta$ is an arbitrary positive number) and write $K_{i j}$ in the form

$$
K_{i j}(s, \tau)=K_{i j}^{(1)}(s, \tau)+K_{i j}^{(2)}(s, \tau), \quad 0 \leq s<\tau<t \leq T,
$$

where

$$
\begin{gathered}
K_{i j}^{(1)}(s, \tau)=-d_{i}(s) N_{j, \delta}(s, \tau), K_{i j}^{(2)}(s, \tau)=d_{i}(s)\left((-1)^{j} q_{j}(s) \frac{\partial Z_{j 1}(s, 0, \tau, 0)}{\partial x}-\right. \\
-\frac{\gamma(s)}{2} G_{j}(s, 0, \tau, 0)-\int_{D_{j}^{\delta}}\left[Z_{j 1}(s, 0, \tau, 0)-Z_{j 1}(s, y, \tau, 0)\right] \mu(s, d y)- \\
\left.-\int_{D_{j} \backslash D_{j}^{\delta}}\left[G_{j}(s, 0, \tau, 0)-G_{j}(s, y, \tau, 0)\right] \mu(s, d y)+(-1)^{i+j} \frac{q_{3-i}(s)}{\sqrt{b_{3-i}(s, 0)}} \tilde{K}_{j}(s, \tau)\right) .
\end{gathered}
$$

By the inequalities (9), (12), we estimate each of the five terms on the right hand side of the expression for $K_{i j}^{(2)}$. Especially for the difference $Z_{j 1}(s, 0, \tau, 0)-Z_{j 1}(s, y, \tau, 0)$ we additionally use the finite-increments formula with respect to the variable $y$. We obtain

$$
\left|K_{i j}^{(2)}(s, \tau)\right| \leq c(\delta)(\tau-s)^{-1+\frac{\alpha}{2}}
$$

where $c(\delta)$ is some positive constant, depending on $\delta$.

Proceeding by the same considerations, using the inequalities (9) and the Lagrange formula for the difference $Z_{j 0}(s, 0, \tau, 0)-Z_{j 0}(s, y, \tau, 0)$, we can also estimate the function $K_{i j}^{(1)}(s, \tau)$. However, on the right hand side of the estimation for $K_{i j}(s, \tau)$, in contrast to $(30)$, the factor $(\tau-s)^{-1}$ appears. This means that the function $K_{i j}^{(1)}(s, \tau)$, and thus, the function $K_{i j}(s, \tau)$, has non-integrable singularity. Nevertheless, we show that the method of successive approximations can be applied to the system of integral equations (24). Indeed, using the representation (29) as well as the inequalities (27) and (30) by the mathematical induction method, we prove that for the terms of series (25) the following inequality is valid $(0 \leq s<t \leq T)$ :

$$
\left|V_{i}^{(n)}(s, t, \varphi)\right| \leq c\|\varphi\|(t-s)^{-\frac{1}{2}} \sum_{k=0}^{n} C_{n}^{k} \cdot a^{(n-k)} M(\delta)^{k}, \quad n=0,1,2, \ldots
$$

where 


$$
\begin{aligned}
& a^{(k)}=\frac{\left(2 c(\delta) T^{\frac{\alpha}{2}} \Gamma\left(\frac{\alpha}{2}\right)\right)^{k} \cdot \Gamma\left(\frac{1}{2}\right)}{\Gamma\left(\frac{1+k \alpha}{2}\right)}, k=0,1,2, \ldots, n, \\
& M(\delta)=\frac{B}{b} \max _{s \in[0, T]} \int_{D_{1}^{\delta} \cup D_{2}^{\delta}}|y| \mu(s, d y) .
\end{aligned}
$$

Fix $\delta=\delta_{0}$ such that $M\left(\delta_{0}\right)<1$. Then in view of (31), we have $(i=1,2)$

$$
\sum_{n=0}^{\infty}\left|V_{i}^{(n)}(s, t, \varphi)\right| \leq c\|\varphi\|(t-s)^{-\frac{1}{2}} \sum_{n=0}^{\infty} \frac{\left(\frac{c\left(\delta_{0}\right)}{1-M\left(\delta_{0}\right)} T^{\frac{\alpha}{2}} \Gamma\left(\frac{\alpha}{2}\right)\right)^{n}}{\Gamma\left(\frac{1+n \alpha}{2}\right)} \cdot \frac{\Gamma\left(\frac{1}{2}\right)}{1-M\left(\delta_{0}\right)} .
$$

The inequality (32) ensures the convergence of series (25) in $s \in[0, t)$ and gives for $V_{i}(s, t, \varphi), i=1,2$, the estimation

$$
\left|V_{i}(s, t, \varphi)\right| \leq c\|\varphi\|(t-s)^{-\frac{1}{2}}, \quad 0 \leq s<t \leq T .
$$

From estimations (9), (12) and (33), it follows that the simple-layer potential in (20) exists and satisfies the initial condition $\lim _{s \uparrow t} u_{i 1}(s, x, t)=0$ and the inequality (16) with $r=p=0$. This means that the function $u(s, x, t), 0 \leq s<t \leq T, x \in \mathbb{R}$, defined by relations (20), (25) is the desired solution of the problem (5)-(8), which belongs to class (18) and satisfies the inequality (19).

The uniqueness of the solution $u(s, x, t)$ follows from the maximum principle for parabolic equations (see [26, Ch. II]).

The proof of the Theorem 3.1 is now complete.

Consider the two-parameter family of linear operators $T_{s, t}, 0 \leq s<t \leq T$, acting on the function $\varphi \in C_{b}(\mathbb{R})$ by the rule

$$
T_{s, t} \varphi(x)=u(s, x, t, \varphi),
$$

where $u(s, x, t, \varphi)$ is the solution of the problem (5)-(8) with function $\varphi$ in (6).

Let us show that the family of operators $T_{s, t}$ is the desired semigroup. To this end, note first that operators $T_{s, t}$ have the following property: if the sequence $\varphi_{n} \in C_{b}(\mathbb{R})$ is such that $\lim \varphi_{n}(x)=\varphi(x)$ for all $x \in \mathbb{R}$ and, in addition, $\sup _{n}\left\|\varphi_{n}\right\|<\infty$, then $\lim _{n \rightarrow \infty} T_{s, t} \varphi_{n}(x)=T_{s, t} \varphi(x)$ for all $0 \leq s<t \leq T, x \in \mathbb{R}$. The proof of this property is based on well-known assertions of calculus on passage of the limit under the summation and integral signs (here this concerns series (25) 
and integrals on the right side of equality (20)). This property allows us to prove the next properties of the operator family $T_{s, t}$, without loss of generality, under the assumption that the function $\varphi$ has compact support.

Now we prove that the operators $T_{s, t}, 0 \leq s<t \leq T$, are positive (a positive operator is one that maps nonnegative functions to nonnegative functions).

Lemma 3.1. If the function $\varphi \in C_{b}(\mathbb{R})$ is nonnegative for all $x \in \mathbb{R}$, then $T_{s, t} \varphi(x)$ is also nonnegative for all $0 \leq s<t \leq T, x \in \mathbb{R}$.

Proof. Let $\varphi$ be any nonnegative function in $C_{b}(\mathbb{R})$ with a compact support. Denote by $m$ the minimum of $T_{s, t} \varphi(x)$ in $(s, x) \in[0, T] \times \mathbb{R}$ and assume that $m<0$. From the minimum principle it follows that there exists $s_{0} \in(0, t)$ such that $T_{s_{0}, t} \varphi(0)=m$. But then the inequalities

$$
\begin{aligned}
& q_{1}\left(s_{0}\right) \frac{\partial T_{s_{0}, t} \varphi(0-)}{\partial x} \leq 0, \quad q_{2}\left(s_{0}\right) \frac{\partial T_{s_{0}, t} \varphi(0+)}{\partial x} \geq 0, \\
& \gamma\left(s_{0}\right) T_{s_{0}, t} \varphi(0) \leq 0, \quad \int_{D_{1} \cup D_{2}}\left(T_{s_{0}, t} \varphi(0)-T_{s_{0}, t} \varphi(y)\right) \mu\left(s_{0}, d y\right) \leq 0
\end{aligned}
$$

must hold. Furthermore, Theorem 14 in [26, Ch. II, Sec. 5] assures us that

$$
\frac{\partial T_{s_{0}, t} \varphi(0-)}{\partial x}<0, \quad \frac{\partial T_{s_{0}, t} \varphi(0+)}{\partial x}>0 .
$$

Next, since $q_{1}\left(s_{0}\right)+q_{2}\left(s_{0}\right)>0$, it becomes clear that in the case of $s=s_{0}$ the fulfillment of the conjugation condition (8) is impossible. The contradiction we arrived at indicates that $m \geq 0$, so the lemma is proved.

By similar considerations to those in proof of Lemma 3.1, it can be easily verified that the operators $T_{s, t}$ are contractive, i.e.,

$$
\left\|T_{s, t}\right\| \leq 1, \quad 0 \leq s<t \leq T
$$

Finally, we show that the operator family $T_{s t}$ has a semigroup property

$$
T_{s, t}=T_{s, \tau} T_{\tau, t}, \quad 0 \leq s<\tau<t \leq T .
$$

This property is a consequence of the assertion of uniqueness of the solution of the problem (5)-(8). Indeed, to find $u(s, x, t)=T_{s, t} \varphi(x)$ when $\lim _{s \uparrow t} u(s, x, t)=\varphi(x)$, the problem (5)-(8) can be solved first in the time interval $[\tau, t]$ and then in the time interval $[s, \tau]$ with the "initial" function $u(\tau, x, t)=T_{\tau, t} \varphi(x)$ which was obtained. In other words, $T_{s, t} \varphi(x)=T_{s, \tau}\left(T_{\tau, t} \varphi\right)(x), \varphi \in C_{b}(\mathbb{R})$, or $T_{s, t}=T_{s, \tau} T_{\tau, t}$.

The above properties of operators $T_{s, t}$ imply the following assertion (see [1, Ch. II, Sec. 1]): 
Theorem 3.2. Let the conditions of Theorem 3.1 hold. Then the two-parameter semigroup of operators $T_{s, t}, 0 \leq s<t \leq T$, defined by formula (34), describes the inhomogeneous Feller process in $\mathbb{R}$, which coincides in $D_{1}$ and $D_{2}$ with the diffusion processes generated by $A_{s}^{(1)}$ and $A_{s}^{(2)}$, respectively, and its behavior at the point zero is determined by the conjugation condition (4).

Let $P(s, x, t, d y)$ be the transition probability of process corresponding to semigroup $T_{s, t}, 0 \leq s<t \leq T$. Then, for any $\varphi \in C_{b}(\mathbb{R})$,

$$
T_{s, t} \varphi(x)=\int_{\mathbb{R}} P(s, x, t, d y) \varphi(y) .
$$

The integral representation of the semigroup $T_{s, t}$, we derived, is useful in the study of the behavior of the corresponding Markov process. We may assert for instance that if the measure $\mu$, which corresponds to the jump-like exit of the process from the point zero, satisfies the additional conditions

$$
\int_{D_{i}} y \mu(s, d y) \in C([0, T]), \quad \int_{D_{i}} y^{2} \mu(s, d y) \in C([0, T]),
$$

then the transition probability $P(s, x, t, d y)$ satisfies the relations:

$$
\begin{aligned}
& \lim _{\Delta s \downarrow} \int_{\mathbb{R}} \varphi(x)\left(\frac{1}{\Delta s} \int_{\mathbb{R}}(y-x) P(s, x, s+\Delta s, d y)\right) d x=\int_{\mathbb{R}} a(s, x) \varphi(x) d x+a_{0}(s) \varphi(0), \\
& \lim _{\Delta s \downarrow 0} \int_{\mathbb{R}} \varphi(x)\left(\frac{1}{\Delta s} \int_{\mathbb{R}}(y-x)^{2} P(s, x, s+\Delta s, d y)\right) d x=\int_{\mathbb{R}} b(s, x) \varphi(x) d x+b_{0}(s) \varphi(0),
\end{aligned}
$$

where $\varphi$ is any continuous function with a compact support,

$$
\begin{aligned}
& a(s, x)=\left\{\begin{array}{l}
a_{i}(s, x), s \in[0, T], x \in D_{i}, i=1,2, \\
\sum_{i=1}^{2} l_{i}(s) a_{i}(s, 0), s \in[0, T], x=0,
\end{array}\right. \\
& b(s, x)=\left\{\begin{array}{l}
b_{i}(s, x), s \in[0, T], x \in D_{i}, i=1,2, \\
\sum_{i=1}^{2} l_{i}(s) b_{i}(s, 0), s \in[0, T], x=0,
\end{array}\right. \\
& a_{0}(s)=\frac{1}{2}\left(d_{1}(s)+d_{2}(s)\right)\left(q_{2}(s)-q_{1}(s)+\int_{D_{1} \cup D_{2}} y \mu(s, d y)\right) \text {, } \\
& b_{0}(s)=\frac{1}{2}\left(d_{1}(s)+d_{2}(s)\right) \int_{D_{1} \cup D_{2}} y^{2} \mu(s, d y), \quad l_{i}(s)=\frac{q_{i}(s) \sqrt{b_{3-i}(s, 0)}}{q_{1} \sqrt{b_{2}(s, 0)}+q_{2} \sqrt{b_{1}(s, 0)}} .
\end{aligned}
$$


One can prove these relations by direct calculations upon using (13), (14).

The relations (36), (37) means, in particular, that in case the constructed process is continuous, it can be treated as a generalized diffusion process in the sense of Portenko [3,4] with the diffusion coefficient $b(s, x)+b_{0}(s) \delta(x)$ and the drift coefficient $a(s, x)+a_{0}(s) \delta(x)$, where $\delta(x)$ is the Dirac $\delta$-function. This completes the proof of the following theorem:

Theorem 3.3. Assume that the conditions of Theorem 3.1 and the condition (35) hold. Then the inhomogeneous Feller process corresponding to the semigroup $T_{s, t}, 0 \leq s<t \leq T$, defined by formula (34) is a generalized diffusion process whose transition probability $P(s, x, t, d y)$ satisfies the relations (36), (37).

\section{Process with properties of partial reflection, absorption and jump-like exit from a sticky boundary}

The purpose of this section is to investigate the problem (5)-(8) when the conjugation condition of Feller-Wentzell (8) is general, in sense, that it can include all its five terms. In this case, the common boundary $S=\{0\}$ of the domains $D_{1}$ and $D_{2}$ has one more property: it is "sticky", i.e.,

II. $r(s)>0$ for all $s \in[0, T]$.

The existence of the desired semigroup is established under the following additional assumptions:

4.1. The function $r$ is Hölder continuous with exponent $\frac{\alpha}{2}$ on $[0, T]$.

4.2. For any function $f \in C_{b}(\mathbb{R})$ and any $\delta>0$ the integrals

$$
\int_{D_{i}^{\delta}} y f(y) \mu(s, d y), \quad \int_{D_{i} \backslash D_{i}^{\delta}} f(y) \mu(s, d y), \quad i=1,2,
$$

are Hölder continuous with exponent $\frac{\alpha}{2}$ in $s \in[0, T]$.

As in previous section, we find a solution of the problem (5)-(8) of the form (20) with the unknown functions $V_{i}$ to be determined. First we note that in view of relations (5)-(7), the condition $(8)$ reduces to

$$
u(s, 0, t)=\varphi(0)-\int_{s}^{t} g(\tau, t) d \tau,
$$

where

$$
\begin{aligned}
g(\tau, t) & =\frac{1}{r(\tau)}\left(q_{1}(\tau) \frac{\partial u(\tau, 0-, t)}{\partial x}-q_{2}(\tau) \frac{\partial u(\tau, 0+, t)}{\partial x}+\gamma(\tau) u(\tau, 0, t)+\right. \\
& \left.+\int_{D_{1} \cup D_{2}}[u(\tau, 0, t)-u(\tau, y, t)] \mu(\tau, d y)\right) .
\end{aligned}
$$


Then, substituting (20) into both sides of (38), we obtain, upon using the relation (17), the system of Volterra integral equations of the first kind

$$
\Lambda_{i}(s, t, \varphi)=\int_{s}^{t} G_{i}(s, 0, \tau, 0) V_{i}(\tau, t, \varphi) d \tau+\sum_{j=1}^{2} \int_{s}^{t} P_{j}(s, \tau) V_{j}(\tau, t, \varphi) d \tau, \quad i=1,2,
$$

where

$$
\begin{gathered}
P_{j}(s, \tau)=\frac{q_{j}(\tau)}{r(\tau) b_{j}(\tau, 0)}-\int_{s}^{\tau} \frac{\bar{K}_{j}(\rho, \tau)}{r(\rho)} d \rho, \\
\Lambda_{i}(s, t, \varphi)=\varphi(0)-u_{i 0}(s, 0, t)+\int_{s}^{t} \frac{\Psi(\rho, t, \varphi)}{r(\rho)} d \rho,
\end{gathered}
$$

and the functions $\bar{K}_{j}$ and $\Psi$ are the same as in (21).

Applying the transform (22) to both sides of each equation in (39), we get an equivalent system of Volterra integral equations of the second kind

$$
V_{i}(s, t, \varphi)=\sum_{j=1}^{2} \int_{s}^{t} R_{i j}(s, \tau) V_{j}(\tau, t, \varphi) d \tau+\Delta_{i}(s, t, \varphi), \quad i=1,2,
$$

where

$$
\begin{aligned}
& R_{i i}(s, \tau)= \sqrt{\frac{2 b_{i}(s, 0)}{\pi}}\left[\sqrt{\frac{\pi}{2}} \tilde{K}_{i}(s, \tau)-\frac{q_{i}(\tau)}{r(\tau) b_{i}(\tau, 0)}(\tau-s)^{-\frac{1}{2}}-\int_{s}^{\tau}(\rho-s)^{-\frac{1}{2}} \frac{\bar{K}_{i}(\rho, \tau)}{r(\rho)} d \rho\right] \\
& R_{i j}(s, \tau)=-\sqrt{\frac{2 b_{i}(s, 0)}{\pi}}\left[\frac{q_{j}(\tau)}{r(\tau) b_{j}(\tau, 0)}(\tau-s)^{-\frac{1}{2}}+\int_{s}^{\tau}(\rho-s)^{-\frac{1}{2}} \frac{\bar{K}_{j}(\rho, \tau)}{r(\rho)} d \rho\right], \quad i \neq j, \\
& \Delta_{i}(s, t, \varphi)=\sqrt{\frac{2 b_{i}(s, 0)}{\pi}}\left[\frac{1}{2} \int_{s}^{t}(\rho-s)^{-\frac{3}{2}}\left(u_{i 0}(\rho, 0, t)-u_{i 0}(s, 0, t)\right) d \rho-\right. \\
&\left.-\left[u_{i 0}(s, 0, t)-\varphi(0)\right](t-s)^{-\frac{1}{2}}+\int_{s}^{t}(\rho-s)^{-\frac{1}{2}} \frac{\Psi(\rho, t, \varphi)}{r(\rho)} d \rho\right],
\end{aligned}
$$

and the functions $\tilde{K}_{i}, i=1,2$, are the same as in (23).

We now show that the functions $\Delta_{i}$ and the kernels $R_{i j}$ in (43) satisfy the inequalities

$$
\begin{gathered}
\left|\Delta_{i}(s, t, \varphi)\right| \leq c\|\varphi\|(t-s)^{-\frac{1}{2}}, \\
\left|R_{i j}(s, \tau)\right| \leq c(\tau-s)^{-1+\frac{\alpha}{2}} .
\end{gathered}
$$


To prove (41) it suffices to note that the first term as well as the function $\Psi$ on the right hand side of the expression for $\Delta_{i}$ are the parts of the formula for $\Psi_{i}$ in (24).

In order to establish the estimation (42), we first consider the integral term that is a part of the expression for $R_{i j}$, and more precisely, we consider its component

$$
L_{j}(s, \tau)=\int_{s}^{\tau}(\rho-s)^{-\frac{1}{2}} \frac{N_{j, \delta}(\rho, \tau)}{r(\rho)} d \rho,
$$

that has a stronger singularity than the other components of this integral. Here the function $N_{j, \delta}$ is the part of the formula for $\bar{K}_{j}$ and is determined by (28).

We write the integral $L_{j}$ in the form

$$
L_{j}(s, \tau)=L_{j}^{(1)}(s, \tau)+L_{j}^{(2)}(s, \tau)
$$

where

$$
\begin{aligned}
& L_{j}^{(1)}(s, \tau)=\frac{1}{r(\tau)} \int_{s}^{\tau}(\rho-s)^{-\frac{1}{2}} d \rho \int_{D_{j}^{\delta}}\left[Z_{j 0}(\rho, 0, \tau, 0)-Z_{j 0}(\rho, y, \tau, 0)\right] \mu(\tau, d y) . \\
& L_{j}^{(2)}(s, \tau)=\int_{s}^{\tau}(\rho-s)^{-\frac{1}{2}} d \rho \int_{D_{j}^{\delta}}\left[Z_{j 0}(\rho, 0, \tau, 0)-Z_{j 0}(\rho, y, \tau, 0)\right]\left(\frac{\mu(\rho, d y)}{r(\rho)}-\frac{\mu(\tau, d y)}{r(\tau)}\right) .
\end{aligned}
$$

Using the relation

$$
Z_{j 0}(\rho, 0, \tau, 0)-Z_{j 0}(\rho, y, \tau, 0)=\int_{0}^{1}\left[2 \pi b_{j}(\tau, 0)(\tau-\rho)\right]^{-\frac{1}{2}} \frac{\partial}{\partial \theta} e^{\frac{-y^{2} \theta}{2 b_{j}(\tau, 0) \cdot(\tau-\rho)}} d \theta,
$$

we can estimate $L_{j}^{(1)}(s, \tau)$ :

$$
\begin{aligned}
& \left|L_{j}^{(1)}(s, \tau)\right| \leq \frac{1}{\sqrt{2 \pi b}}\left|\int_{s}^{\tau}(\rho-s)^{-\frac{1}{2}}(\tau-\rho)^{-\frac{1}{2}} d \rho \int_{D_{j}^{\delta}} \mu(\tau, d y) \int_{0}^{1} \frac{\partial}{\partial \theta} e^{\overline{2 b \cdot(\tau-\rho)}} d \theta\right|= \\
& =\frac{1}{2 b \sqrt{2 \pi b}}\left|\int_{D_{j}^{\delta}} y \mu(\tau, d y) \int_{0}^{1} y e^{\frac{-y^{2} \theta}{2 b \cdot(\tau-s)}} d \theta \int_{s}^{\tau}(\rho-s)^{-\frac{1}{2}}(\tau-\rho)^{-\frac{3}{2}} e^{\frac{-y^{2} \theta}{2 b \cdot(\tau-s)} \frac{\rho-s}{\tau-\rho}} d \rho\right|= \\
& =\frac{1}{2 b \sqrt{2 \pi b}(\tau-s)}\left|\int_{D_{j}^{\delta}} y \mu(\tau, d y) \int_{0}^{1} y e^{\frac{-y^{2} \theta}{2 b \cdot(\tau-s)}} d \theta \int_{0}^{\infty} z^{-\frac{1}{2}} e^{\frac{-y^{2} \theta}{2 b \cdot(\tau-s)} \cdot z} d z\right| \leq c(\tau-s)^{-\frac{1}{2}} .
\end{aligned}
$$


Consider the integral $L_{j}^{(2)}(s, \tau)$. Applying the Lagrange formula to the difference $Z_{j 0}(\rho, 0, \tau, 0)-Z_{j 0}(\rho, y, \tau, 0)$, we get

$$
\begin{gathered}
L_{j}^{(2)}(s, \tau)=\left.\int_{s}^{\tau}(\rho-s)^{-\frac{1}{2}} d \rho \int_{D_{j}^{\delta}} y \frac{\partial Z_{j 0}(\rho, x, \tau, 0)}{\partial x}\right|_{x=\theta y}\left(\frac{\mu(\tau, d y)}{r(\tau)}-\frac{\mu(\rho, d y)}{r(\rho)}\right)= \\
=\left.\int_{S}^{\tau}(\rho-s)^{-\frac{1}{2}}(\tau-\rho)^{-1} d \rho \int_{D_{j}^{\delta}} y(\tau-\rho) \frac{\partial Z_{j 0}(\rho, x, \tau, 0)}{\partial x}\right|_{x=\theta y}\left(\frac{\mu(\tau, d y)}{r(\tau)}-\frac{\mu(\rho, d y)}{r(\rho)}\right),
\end{gathered}
$$

where $\theta$ is some positive number from the interval $(0,1)$.

Note that $f_{\tau, \rho}^{(j)}(y)=\left.(\tau-\rho) \frac{\partial Z_{j 0}(\rho, x, \tau, 0)}{\partial x}\right|_{x=\theta y}, j=1,2$, as functions of $y$, belong to $C_{b}(\mathbb{R})$ for all $0 \leq s<\rho<\tau<t \leq T$, and they are bounded by some common constant (see (9) when $r=0, p=1$ ). Hence, by the uniform boundedness principle,

$$
\int_{D_{j}^{\delta}} y f_{\tau, \rho}^{(j)}(y)\left(\frac{\mu(\tau, d y)}{r(\tau)}-\frac{\mu(\rho, d y)}{r(\rho)}\right) \leq c(\tau-\rho)^{\frac{\alpha}{2}}
$$

and hence

$$
\left|L_{j}^{(2)}(s, \tau)\right| \leq c(\tau-s)^{-\frac{1}{2}+\frac{\alpha}{2}}, \quad 0 \leq s<\rho<t \leq T .
$$

Combining (44) and (45), we find that

$$
\left|L_{j}(s, \tau)\right| \leq c(\tau-s)^{-\frac{1}{2}}
$$

It is clear that the inequality (46) is also valid for the integral

$$
\int_{s}^{\tau}(\rho-s)^{-\frac{1}{2}} \frac{\bar{K}_{j}(\rho, \tau)}{r(\rho)} d \rho
$$

in the expression for $R_{i j}(s, \tau)$ in (40). Therefore, recalling the estimation

$$
\left|\tilde{K}_{j}(s, \tau)\right| \leq c\|\varphi\|(\tau-s)^{-1+\frac{\alpha}{2}}
$$

we conclude that (42) holds.

From (41) and (42) it follows that the system of integral equations (40) has a solution that can be obtained by the method of successive approximations. 
Besides, the functions $V_{i}(s, t, \varphi), i=1,2$, are continuous in $s \in[0, t)$ and for them the inequality (33) is valid.

Thus we have proved that there exists a classical solution of the parabolic problem of conjugation (5)-(8), which is represented in the form (20) and satisfies the inequality (19). Its uniqueness follows from the maximum principle.

Proceeding in the same way as in Section 3, one can prove that the operator family $T_{s, t} \varphi(x)=u(s, x, t, \varphi), \varphi \in C_{b}(\mathbb{R})$, where $u(s, x, t, \varphi)$ is the solution of the problem (5)-(8) with function $\varphi$ in (6), is the contractive Feller semigroup, which describes the inhomogeneous Markov process in $\mathbb{R}$, such that in the domains $D_{1}$ and $D_{2}$ it coincides with the diffusion processes generated by $A_{s}^{(1)}$ and $A_{s}^{(2)}$, respectively, and its behavior at zero is determined by the conjugation condition (4) for which the condition II certainly holds. If we further assume that the jump measure $\mu$, satisfies the additional condition (35), then by the direct calculations we find that for the transition probability of the constructed process (we denote it by $P(s, x, t, d y))$ the following relations hold:

$$
\begin{aligned}
& \lim _{\Delta s \downarrow 0} \frac{1}{\Delta s} \int_{\mathbb{R}}(y-x) P(s, x, s+\Delta s, d y) d x=a(s, x), \\
& \lim _{\Delta s \downarrow 0} \frac{1}{\Delta s} \int_{\mathbb{R}}(y-x)^{2} P(s, x, s+\Delta s, d y) d x=b(s, x), \\
& a(s, x)=\left\{\begin{array}{c}
a_{i}(s, x), s \in[0, T], x \in D_{i}, i=1,2, \\
\frac{1}{r(s)}\left(q_{2}(s)-q_{1}(s)+\int_{D_{1} \cup D_{2}} y \mu(s, d y)\right), s \in[0, T], x=0,
\end{array}\right. \\
& b(s, x)=\left\{\begin{array}{l}
b_{i}(s, x), s \in[0, T], x \in D_{i}, i=1,2, \\
\frac{1}{r(s)} \int_{D_{1} \cup D_{2}} y^{2} \mu(s, d y), s \in[0, T], x=0,
\end{array}\right.
\end{aligned}
$$

Relations (47), (48) mean, in particular, that if the constructed process with the transition probability $P(s, x, t, d y)$ is continuous, then for this process the Kolmogorov local characteristics exist in an ordinary sense. Furthermore, the drift and diffusion coefficients coincide with the functions $a(s, x)$ and $b(s, x)$, respectively.

The result, obtained in the present section, can be stated in the form of the following theorem:

Theorem 4.1. Let the conditions II, 1.1, 2.1-2.3 and 4.1, 4.2 hold. Then the assertions of Theorems 3.1 and 3.2 are true. If, in addition, the condition (35) holds, then the transition probability $P(s, x, t, d y)$ of the inhomogeneous Feller process, which corresponds to the semigroup $T_{s, t}, 0 \leq s<t \leq T$, defined by (34), satisfies the relations (47), (48). 


\section{Process with absorption and jump properties}

In this section we consider the problem (5)-(8) in the case when the condition (8) is non-transversal, i.e.,

III. $r(s)=q_{1}(s)=q_{2}(s)=0, \gamma(s) \geq 0, \mu\left(s, D_{1} \cup D_{2}\right)>0$ for all $s \in[0, T]$.

In addition, we assume that:

5.1. The function $\gamma$ is Hölder continuous with exponent $\frac{1+\alpha}{2}$ on $[0, T]$.

$5.2 \mu\left(s, D_{1} \cup D_{2}\right)=1$ for all $s \in[0, T]$.

5.3. For any function $f \in C_{b}(\mathbb{R})$ the integrals

$$
\int_{D_{i}} f(y) \mu(s, d y), \quad i=1,2,
$$

are Hölder continuous with exponent $\frac{1+\alpha}{2}$ in $s \in[0, T]$.

Note that in this case the desired operator semigroup $T_{s, t}, 0 \leq s<t \leq T$, exists in the subspace of $C_{b}(\mathbb{R})$ which consists of all $\varphi \in C_{b}(\mathbb{R})$ with $L_{t} \varphi(0)=0$. We denote this subspace by $C_{L}(\mathbb{R})$. This result can be obtained by the scheme similar to that used in two previous sections. We therefore restrict ourselves only to the formulation of the corresponding theorem. The interested reader is referred to [24].

Theorem 5.1. Let the conditions III, 2.1-2.3 and 5.1-5.3 hold. Then we have the following two assertions:

1. For any function $\varphi \in C_{L}(\mathbb{R})$ the problem (5)-(8) has the unique solution which belongs to the class (18). Furthermore, this solution satisfies the inequality (19) and has the form (20).

2. The two-parameter operator semigroup $T_{s, t}, 0 \leq s<t \leq T$, defined on $C_{L}(\mathbb{R})$ by (35), describes the inhomogeneous Feller process in $\mathbb{R}$, which coincides in $D_{1}$ and $D_{2}$ with the diffusion processes generated by $A_{s}^{(1)}$ and $A_{s}^{(2)}$, respectively, and its behavior at the point zero is determined by the conjugation condition (4).

\section{Conclusions}

This chapter is devoted to some questions of the theory of one-dimensional generalized diffusion processes and the theory of parabolic differential equations with nonlocal Feller-Wentzell boundary conditions. The most general classes of generalized diffusion processes appear in the problem of pasting together two diffusion processes on a line. In our formulation, this problem is to construct the two-parameter Feller semigroup associated with the inhomogeneous Markov process on a line which coincides, in the interior points of the half-lines separated 
from each other by a fixed point, with the ordinary diffusion processes given there, and its behavior at the common boundary of these domains is determined by the Feller-Wentzell conjugation condition which has a nonlocal character. In a general case, this condition includes the values of the function and its derivatives up to the second order inclusive on the boundary and also the integral over the union of these domains. There are two classes of nonlocal conditions: transversal and non-transversal. In the transversal case, the orders of nonlocal terms are less than the orders of local ones while in the non-transversal case they are equal. The results of investigation of the both cases in the problem of pasting together two diffusion processes on a line are presented in this chapter.

In order to solve the problems formulated here, authors use the analytical methods. Such an approach allows one to define the desired operator family using the solution of the corresponding conjugation problem for a linear parabolic equation with discontinuous coefficients, to which the output problem is reduced. A classical solvability of this problem is established by the boundary integral equations method under some conditions on its output data. It is shown that the twoparameter Feller semigroup constructed in this way corresponds to the desired process.

\section{References}

[1] Dynkin E.B., Markov Processes, Academic Press, New York, Springer, Berlin 1965.

[2] Ikeda N., Watanabe S., Stochastic Differential Equations and Diffusion Processes, North Holland Publ. Co., Amsterdam-Oxford-New York 1986.

[3] Portenko M.I., Generalized Diffusion Processes, Naukova Dumka, Kyiv 1982; Amer. Math. Soc., Providence, RI, 1990.

[4] Portenko M.I., Diffusion Processes in Media with Membranes, Institute of Mathematics of the NAS of Ukraine, Kyiv 1995.

[5] Kopytko B.I., Portenko M.I., The problem of pasting together two diffusion processes and classical potentials, Theory Stochast. Processes 2009, 15(31), 2, 126-139.

[6] Wentzell A.D., On boundary conditions for multidimensional diffusion processes, Theory Prob. Appl. 1959, 2, 5, 172-185.

[7] Feller W., The parabolic differential equations and associated semigroups of transformations, Ann. Math. 1952, 55, 468-518.

[8] Wentzell A.D., Semigroups of operators that correspond to a generalized differential operator of second order, Dokl. Akad. Nauk SSSR 1956, 111, 2, 269-272.

[9] Langer H., Schenk W., Knotting of one-dimensional Feller process, Math. Nachr. 1983, 113, 151-161.

[10] Petruk O., Kopytko B., Time-dependent shock acceleration of particles. Effect of the timedependent injection, with application to supernova remnants, Monthly Notices of the Royal Astronomical Society 2016, 462, 3104-3114.

[11] Skubachevskii A.L., Nonlocal elliptic problems and multidimensional diffusion processes, Russian J. Math. Phys. 1995, 3, 3, 327-360.

[12] Taira K., On the existence of Feller semigroups with boundary conditions, Mem. Amer. Math. Soc. 1992, 99, 475, 1-65. 
[13] Anulova S.V., Diffusion processes: discontinuous coefficients, degenerate diffusion, randomized drift, Dokl. Akad. Nauk SSSR 1981, 260, 5, 1036-1040.

[14] Kulinich G.L., On the limit behavior of the distribution of the solution of a stochastic diffusion equation, Theory Prob. Appl. 1967, 12, 3, 497-499.

[15] Walsh I.B., A diffusion with discontinuous local time, Société Mathématique de France, Astérisque 1978, 52-53, 37-45.

[16] Harrison J.M, Shepp L.A., On skew Brownian motion, Annals of Probability 1981, 9, 2, 309-313.

[17] Zaitseva L.L., On a multidimensional Brownian motion with partly reflecting membrane on a hyperplane, Theory Stochast. Processes 1999, 5(21), 3-4, 258-262.

[18] Engelbert H.J., Schmidt W., Strong Markov continuous local martingales and solutions of one-dimensional stochastic differential equations, III, Math. Nachr. 1991, 151, 149-197.

[19] Barlow M., Burdzy K., Kaspi H., Mandelbaum A., Variably Skewed Brownian Motion, Electron. Comm. Probab. 2000, 5, 57-66.

[20] Kulik A.M., On the solution of a one-dimensional stochastic differential equation with singular drift coefficient, Ukr. Math. J. 2004, 56, 5, 774-789.

[21] Pilipenko A.Yu., On the Skorokhod mapping for equations with reflection and possible jump-like exit from a boundary, Ukrainian Math. J. 2012, 63, 9, 1415-1432.

[22] Pilipenko A.Yu., An Introduction to Stochastic Differential Equations with Reflection, 1, Universitätsverlag Potsdam, 2014.

[23] Kopytko B.I., Shevchuk R.V., On pasting together two inhomogeneous diffusion processes on a line with the general Feller-Wentzell conjugation condition, Theory Stochast. Processes 2011, $17(33), 2,55-70$.

[24] Shevchuk R.V., Pasting of two one-dimensional diffusion processes, Annales Mathematicae et Informaticae 2012, 39, 225-239.

[25] Ladyzhenskaya O.A., Solonnikov V.A., Ural'tseva N.N., Linear and Quasilinear Equations of Parabolic Type, Amer. Math. Soc., Providence, RI, 1968.

[26] Friedman A., Partial Differential Equations of Parabolic Type, Prentice-Hall, New York 1964. 